\section{Das österreichische Forschungsförderungssystem: Systemevaluiert!}

\author{
Mit der vorliegenden Nummer des tippolicybrief wird eine neue Reihe eröffnet, \\ in der wir in unregelmäßigen Abständen aktuelle nationale und internationale \\ Themen der Technologie-, Innovations- und Forschungspolitik diskutieren \\ und kommentieren. Wir richten uns dabei an ein breites Publikum, wobei \\ politische Entscheidungsträger im Mittelpunkt stehen. Die im tippolicybrief \\ vertretenen Meinungen sind jene der jeweiligen Autorlnnen.
}

Autor

Andreas Schibany

JOANNEUM RESEARCH

Forschungsgesellschaft $\mathrm{mbH}$

Institut für

Technologie- und

Regionalpolitik

Sensengasse 1

1090 Wien, Austria

Tel.: +4315817520-2823

Fax: +4315817520-2820

andreas.schibany@joanneum.at

vie@joanneum.at

www.joanneum.at/rtg

\section{Vorbemerkung}

Die Ergebnisse der mehrere hundert Seiten umfassenden Systemevaluierung der österreichischen Forschungsförderung und -finanzierung ${ }^{1}$ zu kommentieren ist kein leichtes Unterfangen, denn niemals kann ein Kommentar einzelner Teile dem immensen Gesamtaufwand der Systemevaluierung gerecht werden und auf sämtliche Ergebnisse und Empfehlungen eingehen. ${ }^{2}$ Eine kritische Abarbeitung dieser bleibt dem Auftraggeber der Systemevaluierung vorbehalten.

Wenn im folgenden einige Resultate der Systemevaluierung kritisch diskutiert werden, dann geschieht das vor dem Hintergrund des Wissens über die Hürden und Hindernissen dieser Evaluierung - vor allem bezüglich der Datenverfügbarkeit und den diversen politischen Erwartungshaltungen die der Systemevaluierung gegenüber schon im Vorfeld artikuliert wurden.

Eine Evaluierung zeigt aber unseres Erachtens dann die beste Wirkung, wenn sie kommentiert und diskutiert wird und zu Zustimmung und Widerspruch anregt - beim Auftraggeber wie auch in der Experten-Community. Aus dieser Haltung heraus haben wir einige der wichtigsten Schlussfolgerungen der Systemevaluierung herausgegriffen und dort, wo es uns sinnvoll und notwendig erschien, diese kritisch zu diskutieren konträre Positionen formuliert - oder aber auch die affirmativen Einschätzungen unterstrichen.

\section{Zeitpunkt und Verständnis}

Die Systemevaluierung konnte zu keinem besseren Zeitpunkt beauftragt und erstellt werden. Denn vorbei sind die Zeiten in denen man glauben konnte, leicht von anderen Ländern, welche hohe BIP-Wachstumsraten und hohe F\&E-Quoten aufwiesen, Erfolgsrezepte übernehmen zu können - die USA oder Irland waren beliebte Beispiele. Jetzt in der Finanz- und Wirtschaftskrise zeigt sich das Gesicht der wahren Wachstumstreiber und die Bereitschaft, die Governance und Förderstrukturen dieser ehemaligen Vorbildländer zu kopieren ist eher gesunken. Die Systemevaluierung meidet daher konsequenterweise diesen Blick nach außen und unterzieht sich der mühevollen Analyse der österreichspezifischen Verhältnisse. Vielleicht wurde diese Konsequenz auch zu weit getrieben denn auf der Ebene der Instrumente der Forschungsförderung ließen sich schon ganz gute Lehren und Erfahrungen aus anderen Ländern ziehen.

Die Systemevaluierung legt ihrer Analyse einen breiten und umfassenden Ansatz der Innovationspolitik zugrunde. Dieses Grundverständnis von Forschungs-, Technologie- und Innovationspolitik (FTI-Politik) ist zugleich inre größte Stärke, weist es doch auf »die wahrscheinlich größte Herausforderung, die sich aus einer internen Schwäche ergibt«: Österreich hat ein massives Problem im Bildungssystem. Damit bestätigt die Systemevaluierung die Befunde der OECD (2007) oder auch des jüngst erschienen Forschungs- und Technologieberichts: der Bottleneck des österreichischen Innovationssystems liegt

\footnotetext{
1 Die folgenden Ausführungen beziehen sich im Wesentlichen auf den Synthesebericht (Aiginger et al. 2009)

2 Ich danke Wolfgang Polt und Helmut Gassler für Kommentare und Diskussionen.
} 
zur Zeit im Bereich der Humanressourcen. Wie ein roter Faden zieht sich die Argumentation durch die gesamte Systemevaluierung, und kulminiert im letzten Satz des Syntheseberichts: National investment in primary, secondary, and tertiary education provide the foundation of economic development in an innovation led growth regime as it provides a foundation for a portion of the indigenous population to secure advanced education and high-tech work experience. (S. 170).

Heute umfasst die FTI-Politik nicht nur die Politikbereiche, deren Instrumente unmittelbar und ausschließlich auf die Beeinflussung des Innovationsverhaltens oder der Forschungstätigkeit von Unternehmen und öffentlichen Institutionen abzielen. Das Spektrum der von der FTI-Politik berührten Politikbereiche nimmt zu und erhält immer mehr den Charakter eines umfassenden Querschnittsbereichs, der von der Bildungs- über die Wissenschafts-, Technologieund Industriepolitik bis zur Wettbewerbs-, Wirtschafts- (z. B. Steuerrecht, Unternehmensrecht, Kapitalmarktregulierung) und Sozialpolitik (z. B. Arbeitsrecht) reicht und mitunter noch weitere Politikbereiche einbezieht (wie die Migrationspolitik). Genau darin liegt die Notwendigkeit eines Koordinationsmechanismus auf politischer Ebene um dadurch mit einer Stimme im politischen Entscheidungsprozess sprechen zu können. Dieser - durchaus herausragende - Teil über die Rahmenbedingungen verweist sehr deutlich auf diese Notwendigkeit, die letztlich in einer umfassenden Strategie sowie der Etablierung einer hochrangigen Steuerungsgruppe mündet.

\section{Der Blick aufs Ganze}

Mit Blick auf das Ganze des österreichischen Innovationssystems wird - bereits im Titel - > radikaler Wandel < verlangt. Dies wird aus der Notwendigkeit abgeleitet, dass Österreich von einer Follower zu einer Frontrunner Strategie finden müsse. Abgeschlossen ist also der Catching-up Prozess mit seinen inkrementellen Innovationen und Imitationen. Radikale Veränderungen des Innovationssystems müssten entschieden und rasch die Voraussetzungen für die Positionierung Österreichs an der Technologiefront (technological frontier) schaffen.

Die Notwendigkeit einer stärkeren Orientierung an der stechnologischen Grenze < wird hier nicht bestritten. ${ }^{3}$ Hier wird aber eine Dringlichkeit und Radikalität suggeriert, die sich aus den meisten Analysen des österreichischen Innovationssystems über die letzten Jahre so nicht ablesen lassen. Denn auch die ersten Kapitel des Syntheseberichts der Systemevaluierung zeigen eindrucksvoll, wie erfolgreich der Unternehmenssektor im Bereich Innovation und F\&E in den letzten Jahren agiert hat. Hier wird also die jüngste Vergangenheit in einem zu dunklen Schatten gestellt. Denn das immense Exportvolumen sowie die substantielle Verbesserung der Wettbewerbsfähigkeit der österreichschen Unternehmen (ablesbar etwa an der Handelsbilanz, die in den letzten Jahren erstmals in der Nachkriegsgeschichte leichte Überschüsse verzeichnen konnte) kann nicht mit Imitationen und inkrementellen Innovationen alleine erzielt worden sein. Dafür ist die Konkurrenz auf den Weltmärkten schon zu intensiv.

Worin besteht nun die notwendige radikale Veränderung? Was macht die Frontrunner-Strategie aus? Wie muss - neben den notwendigen strukturellen Veränderungen im Ausbildungs- und Wissenschaftssystem - das Fördersystem auf die Herausforderung reagieren, um Österreich ganz vorne zu positionieren? Schon der Titel des Syntheseberichts (Reaching out to the Future Needs Radical Change) schraubt die diesbezügliche Erwartung hoch. Aber hier tut sich ein grundlegender Widerspruch der gesamten Evaluierung auf, denn gegenüber den behaupteten Notwendigkeiten zum , radikalen Wandel < sind die förderpolitischen Empfehlungen im Großen und Ganzen »more of the same «. Einerseits schon bekannter Forderungen nach beispielsweise einer Reduktion von Förderprogrammen, andererseits einer monetären Aufblähung bestehender Förderinstrumente, wie die Erhöhung der Forschungsprämie auf $12 \%$ oder eine Ausweitung der Basisprogramme. Und dass die öffentliche Hand bei der Direktförderung doch mehr radikale und riskante Projekte fördern sollte, steht auch schon lange auf der Agenda. Damit lässt sich vieles Positives erreichen - mit einem radikalen Wandel würde man diese Vorschläge allerdings nur bedingt assoziieren.

\section{Quantitative Ziele für die F \& E-Intensität?}

Spätestens seit der Veröffentlichung der F\&E-Globalschätzung 2009 durch die Statistik Austria, die eine Erhöhung der F\&E-Quote auf 2,73\% für 2009 prognostiziert, sollte die Problematik eines »Quotenziels « deutlich geworden sein. Denn die F\&E-Quote 2009 wird nur deshalb steigen, weil das nominelle BIP sinkt und die gesamten F \&E-Ausgaben gegenüber 2008 um marginale $1,8 \%$ steigen. Nun ist diese Ambivalenz den Autorlnnen der Evaluierung, die gleichzeitig auch die BIP-Prognosen erstellen, natürlich bewusst. Und ein zu enges Verständnis

3 Wenngleich auch in der Systemevaluierung nirgends explizit erläutert wird, wie weit Österreich von der technologischen Grenze noch entfernt ist. Eine thematische Aufbereitung dieser auf Griffith, Aghion und andere zurückgehende Konzepte (in denen im Wesentlich die Totale Faktorproduktiviäten von Industriesektoren miteinander in Beziehung gesetzt werden), sowie deren Relevanz für Österreich, wird Thema eines der folgenden policybriefs sein. 


\section{Das österreichische Forschungsförderungssystem: Systemevaluiert}

eines Quotenziels wird in der Evaluierung auch richtigerweise als » naiv oder zynisch « (S. 133) bezeichnet. Vor dem Hintergrund der Erfahrungen mit Quotenzielen und den Entwicklungen seit dem Jahre 2000 lassen sich folgende kurze Anmerkungen hierzu machen:

- Österreich konnte mit dem Ziel, bis 2010 eine F\&E-Quote von $3 \%$ erreichen zu wollen, eine immense Mobilisierung erreichen. Forschung, Technologie und Innovation standen ganz oben auf der politischen Agenda und die öffentliche Hand stellte zusätzliche Mittel bereit.

- Gleichzeitig bewirkte die ständige Betonung, Österreich befände sich auf dem 3\%-Pfad, auch eine politische Vernachlässigung von anderen forschungsrelevanten Politikbereichen (wie Wettbewerbs-, Arbeitsmarkt- oder Bildungspolitik) oder der Governance der FTI-Politik selbst. Solange nur genügend Geld in Forschung investiert wurde und die F\&E-Quote stieg, sah sich die Politik auf einem Erfolgskurs. Der Preis dafür ist ein Problem in der Governance. Die vorliegende Evaluierung wie auch der CREST-Bericht (2008) machen dies deutlich.

- Hat das 3\%-Ziel vor allem den catching-up Prozess seit Mitte der 1990er Jahre begleitet, so lässt sich nur schwer daraus linear die Notwendigkeit eines , 4 \%-Ziels zur Erreichung einer Position unter den wissenschaftlich und technologisch führenden Ländern (mithin unter den >Frontrunnern «) ableiten. Denn wie sich auch bei hoch entwickelten Volkswirtschaften mit einer hohen F\&E-Intensität zeigen lässt, steigt die F\&E-Quote nicht linear und kontinuierlich. In den USA ist die F\&E-Quote seit Jahren konstant und auch in Schweden oder Finnland gibt es nur noch geringe Veränderungen, wie die folgende Abbildung zeigt.

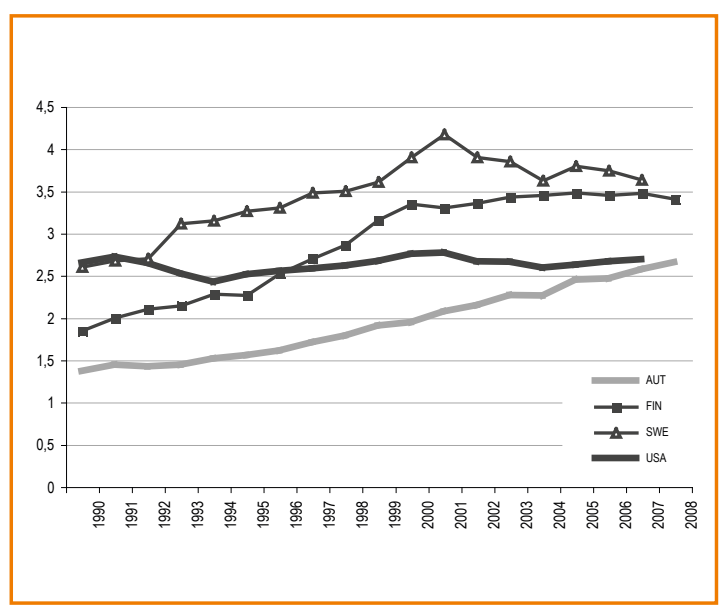

Entwicklung der F\&E-Quoten in ausgewählten Ländern Quelle: OECD (MSTI)

Die F\&E-Intensität sollte unseres Erachtens daher vor allem qualitativ diskutiert werden: welches Ausmaß ist vor dem Hintergrund der Systemperformance Österreichs sinnvoll anzustreben. Ein quantitativ genau definierbares "Gleichgewichtsniveau « für Österreich und ein entsprechendes Steigerungspotential ist nicht wirklich seriös zu bestimmen.

Ökonomisch lässt sich somit nicht wirklich schlüssig begründen, auf welchem Niveau eine effiziente F \& E-Quote in Österreich liegen sollte. Dies wissend und die Literatur zur Internationalisierung von F \& E kennend, verwundert insbesondere die folgende Empfehlung der Evaluierung: From an economic point of view the tax credit should be considerably higher than today in order to keep - and renew - the attractiveness for research in Austria in a more competitive environment and to draw near to the targeted $R \& D$ quotas set by the government (S. 155). Zumindest die Forderung nach 'keep and renew < als Begründung einer Erhöhung der Forschungsprämie klingt nicht wie eine selbstbewusste und ökonomische begründete Frontrunner-Strategie.

\section{Die steuerliche F \& E-Förderung}

Die spezifische Analyse über die Struktur und Effekte der steuerlichen F\&E-Förderung stand im Mittelpunkt der Systemevaluierung und die Erwartungshaltung bezüglich der Ergebnisse und Empfehlungen waren besonders hoch. Erstmals wurde dieses Förderinstrument im Auftrag der öffentlichen Hand einer Evaluierung unterzogen. Aber wer dachte, dass die öffentliche Hand auch die entsprechenden Mikrodaten (mit den gewohnten Vertraulichkeitserklärungen) den Evaluatoren zugänglich macht um dadurch objektive Analysen zu ermöglichen, der irrt. Dass die Bundesministerien eine Systemevaluierung der österreichischen Forschungsförderung beauftragen ohne die vorhandenen und dafür notwendigen Daten zur Verfügungen zu stellen ist perhorreszierend.

Trotz dieser Ärgerlichkeit muss über die Wirkung und Effektivität dieses Förderinstrument nachgedacht werden - ging es doch 2008 um ein Volumen von 340 Mill. $€$ ausbezahlter Forschungsprämie, und für 2009 rechnet die Statistik Austria mit einem Volumen von 380 Mill. $€$.

Auch international ist die steuerliche F\&E-Förderung zu einem wichtigen Instrument geworden: 20 der 30 OECD-Staaten und 15 der 27 EU-Mitgliedsstaaten haben inzwischen eine steuerliche F\&E-Förderung eingeführt. Zu den prinzipiellen Vorzügen dieses indirekten Instruments gehört, dass es nur auf die Forschungsaktivität als solche abzielt, nicht aber auf ihre spezifischen Inhalte. Dadurch beeinflusst der Staat nicht die inhaltliche Ausrichtung der Forschung und Entwicklung, sondern reduziert die Kosten der Durchführung jedweder F\&E-Aktivitäten. Insbesondere durch das gesetzlich festgelegte Anrecht auf die Forschungsprämie (welche mittlerweile fast das gesamte steuerliche F\&E-Fördervolumen abdeckt und gegenwärtig $8 \%$ beträgt) entfallen komplexe Antragsverfahren. 
Die Forschungsprämie kommt einer Barauszahlung gleich und ist für Unternehmen interessant, die von Forschungsfreibeträgen mangels Gewinn (bzw. Steuerschuld) nicht profitieren können. Daher liegt es auf der Hand, dass von dieser Art der Förderung vor allem KMU und junge Start-up Unternehmen profitieren. Dieses Instrument entfaltet somit eine Breitenwirkung, indem es vor allem die Anzahl der geförderten Unternehmen mit F \& E-Aktivitäten maximiert. Da in Österreich die Inanspruchnahme der Forschungsprämie gleichermaßen für alle $\mathrm{F}$ \& E-durchführenden Unternehmen gilt, widerspiegelt die Aufteilung des Fördervolumens auch die realen Ausgabenverhältnisse: fast $70 \%$ der Forschungsprämie entfielen 2006 auf Unternehmen mit mehr als 500 Beschäftigten.

Neben der Breitenwirkung erweist sich die steuerliche F\&E-Förderung auch als sehr flexibles and anpassungsfähiges Instrument, was sich an der Varianz in der konkreten Ausgestaltung in den jeweiligen Ländern erkennen lässt. Auch die Europäische Kommission begrüßte in einer Mitteilung (Europäische Kommission 2006) die steuerliche Stimulierung von F\&E, erkannte aber gleichzeitig die Gefahr, dass die europäische Unternehmenssteuerlandschaft weiter aufsplittert. Dazu wurden in der Mitteilung gewisse Leitlinien in der Gestaltung steuerlicher F\&E-Fördermaßnahmen dargestellt, wie u. a.

- Förderung großer transnationaler F\&E-Projekte

- Junge innovative Unternehmen

- Förderung der Forschungsfinanzierung durch Stiftungen

- Grenzüberschreitende Mobilität von Wissenschaftlern

Vor diesem Hintergrund erweisen sich die Empfehlungen der Systemevaluierung erstaunlich uninspiriert. Zwar reduziert die empfohlene Beschränkung der steuerlichen F\&E-Förderung auf die Forschungsprämie und die Abschaffung der beiden Varianten des Forschungsfreibetrages sicherlich die Komplexität, aber die Begründung einer Erhöhung der Forschungsprämie von $8 \%$ auf $12 \%$ bleibt ein wenig dünn: With respect to the volume-based components, a pareto-optimal redesign of the current structure of fiscal incentives for R\&D is achieved when the premium rate rises to 12.5 per cent of eligible $R \& D$ expenditures. (Teilstudie 4, S. 60). ${ }^{4}$ Und der Synthesebericht sieht die simple Erhöhung einer recht unspezifische Maßnahme als den Inbegriff einer Frontrunner-Strategie: Such policy change is quite in line with the recommended focus shift (i.e. froma catch-up to a frontrunner strategy) (S. 155).

Da präsentierte sich der Rat für Forschung und Technologieentwicklung - der wahrlich kein Feind der Großunternehmen ist - ideenreicher und erkannte das Potential der steuerlichen F\&E-Förderung für junge, forschungsintensive KMU. In der Empfehlung vom 09.12.2008 ist zu lesen:

Der bestehende Prozentsatz von $8 \%$ Forschungsprämie könnte angehoben und entsprechend gespreizt werden, wobei kleinere, jüngere Unternehmen einen höheren Prozentsatz erhalten, ältere, größere Unternehmen einen niedrigeren, da die größte Finanzbelastung gerade in der Anfangsphase liegt. (S. 9)

Auch in anderen Studien (z. B. Schneider et al. 2005) wird eindeutig gezeigt, dass eine deutliche Erhöhung der Forschungsprämie für junge Unternehmen (i) relativ »wenig kostet « und (ii) eine wichtige Unterstützung für junge, innovative Unternehmen (aus dem Dienstleistungssektor) darstellt und deren Performanz stärkt. Die Kompensation für die Deckelung bei Großunternehmen kann in der missionsorientierte Forschung liegen - einer Forschung mit hohem sozialen Ertrag, Langfristigkeit, der Einbindung forschungsintensiver Unternehmen und einer starken Rolle der öffentlichen Hand. Leider finden sich zu dieser Art von Forschung keine Anregungen im Evaluierungsbericht.

Auch ist es bedauerlich, dass die spezielle Situation der KMU - vor allem im Hinblick auf die gegenwärtige Wirtschaftskrise - weitgehend unberücksichtig bleibt. Durch die zunehmende Risikoaversion und der daraus resultierenden Kreditrestriktion stehen vor allem junge Unternehmen vor einem massiven Finanzierungsproblem. Denn in Zeiten politischer Rettungsaktionen konzentriert sich die Aufmerksamkeit der vom Staat gestützten Banken vor allem auf solche Unternehmen, dessen Belegschaft keine Regierung gern auf der Strasse sieht. Junge, innovative Unternehmen tragen nur das Potential großer Innovationen in sich und haben per definitionem wenig Lobbyingeinfluss. Nun soll hier nicht einer prozyklischen Förderung das Wort geredet werden - FTI-Politik eignet sich nur schlecht als Konjunkturpolitik. Aber es darf nicht unerwähnt bleiben, dass vor allem junge, innovative Unternehmen die ersten Opfer eines dysfunktionalen Kreditsystems sind und daher gerade in Abschwungphasen ein innovations- und forschungsstützendes Fördersystem hilfreich ist.

Auch hätte das eine oder andere Interview und Gespräch mit ausgewählten Personen des Unternehmenssektors gezeigt, dass zwar in wirtschaftlich guten Zeiten die aus der Forschungsprämie lukrierten Summen den Forschungsabteilungen zugute kommen. In Zeiten der Rezession liegen andere Notwendigkeiten vor, und die Erhöhung eines sehr unspezifischen Fördervolumens kann dazu führen, dass andere Finanzlöcher damit gestopft werden. Bei der steuerlichen F\&E-Förderung liegen zurzeit wenig Kontrollmöglich-

4 Die wirkliche Empfehlung bleibt ein wenig im Dunkeln. In der Teilstudie 4 (Tax Incentives Schemes for R\&D) wird eine Erhöhung der Forschungsprämie auf 12,5\% empfohlen. Im Synthesebericht wird von $12 \%$ gesprochen, und das Szenario über die zu erwarteten Kosten wird auf der Basis einer Forschungsprämie von $10 \%$ gerechnet. 


\section{Das österreichische Forschungsförderungssystem: Systemevaluiert}

keiten vor. Inwieweit sich das durch die Bildung eines » pool of highly qualified auditors", welche die ex-post Kontrolle übernehmen sollen, ändert, bleibt dahingestellt.

\section{Die Krux mit dem Risiko}

Die unterschiedlichen Ziele, welche mit den Förderinstrumenten verfolgt werden, scheinen für die Systemevaluierung recht klar zu sein: Die steuerlichen Förderung dient der Breitenwirkung, und mit der direkten Förderung sollen vor allem solche Projekte gefördert werden, die einen hohen sozialen Ertrag, hohes Risiko und einen radikalen Innovationsgehalt aufweisen. Begleitet werden die Ziele der Direktförderung mit einer sehr klaren Outputorientierung seitens der Förderagenturen, durch welche diese Art der Förderung abgewickelt wird. Damit soll auch ein besseres Verhältnis zwischen Input- und Outputindikatoren erreicht werden.

Mit diesen Argumenten - die zugegeben etwas verkürzt dargestellt wurden - bewegt sich die Systemevaluierung auf der Ebene der ökonomischen Begründung für staatliches Intervenieren: Dieses ist dann berechtigt, wenn Marktversagen in Form von externen Effekten oder auch in Form eines zu hohen Risikos vorliegt, was letztlich zu einer zu geringen Investitionsneigung seitens der Unternehmen führt. Die Aufgabe des Staates besteht darin, die unterschiedlichen Formen des Marktversagens in jenen Bereichen zu korrigieren, in denen bestimmte technologische Entwicklungen oder Innovationsaktivitäten zu einer Wohlfahrtsteigerung führen. Trotz dieser sauberen Argumentation bestehen Probleme in der Vorstellung ihrer Realisierbarkeit:

Auf Projektebene ist die Förderung von Risiko kaum möglich - das ist Risiko in kurzen Hosen. Risiko ist mit Scheitern verknüpft, und jeder der schon Mitglied in Jurysitzungen war, weiß, dass hier Fragen wie: ökonomisches Potential und Verwertung, Marktrelevanz, konkreter Nutzen der Projektergebnisse, Erhöhung des Innovationsniveaus etc. förderentscheidend sind. Ein Projektantrag wird mit der bisherigen Performanz des Antragstellers in Verbindung gebracht und wenn nur der Funken eines Scheiterns vorliegt, wird das Projekt eher nicht zur Förderung empfohlen. Hier liegt eine mentale und gleichzeitig nachvollziehbare Barriere im Förderverhalten vor.

Das führt zu einem Trade-off zwischen Risikoneigung und Outputsteuerung seitens der Agenturen. Da die Systemevaluierung klare Zielvorgaben seitens der Agenturen als Steuerungsinstrument für die erhöhte Autonomie vorsieht, impliziert das anreizinduziertes Förderverhalten. Und da das Scheitern von Projekten nicht Teil einer Zielvereinbarung sein kann, werden Förderagenturen sich davor hüten, riskante Projekte zu fördern - schließlich wollen die Outputziele ja erreicht werden.
Die stärkere Fokussierung auf Outputs im Förderverhalten sieht die Systemevaluierung als bessere strategische Steuerung und nennt auch einige Beispiele outputbasierter Indikatoren:

The general performance of the innovation system might be assessed with reference to the number of patents and publications, the speed of technology diffusion, competitiveness in high-tech industries and in the highest quality segment of individual industries. More specifically, output goals for a scheme devised to promote innovative start ups may be defined in terms of the number of firms entering a market, the dynamics of employment in new firms, the growth of firms after five years, the number of firms developing new products or innovating continuously. Output goals for a strategy to deepen innovation may be given by the number or size of innovations new to the market, the number of firms which realise successful innovations in an emerging key technology, the number of newly initiated research co-operations with (foreign) universities, the share of patents in the triad (USA-Japan-Europe), revenue accrued from licenses, etc. (Synthesebericht, S. 144)

Und in den Empfehlungen auf der folgenden Seite ist zu lesen, dass dies zu einem besseren Abschneiden Österreichs bei diversen Outputindikatoren führen würde. Gleichzeitig sollen sich die Förderempfänger bewusst sein, "... that their post-funding performance will be increasingly benchmarked against output-based indicators. «(S.145) Bevor jedoch ein solches System implementiert wird, sollte man sich zwei Tatsachen verdeutlichen:

- Abzüglich der Forschungsprämie hat der Staat 2006 von den 4,4 Mrd. € Forschungsausgaben des Unternehmenssektors 272 Mio. € finanziert. Das sind $6,1 \%$. Damit spielt der Staat eine eher untergeordnete Rolle in der Finanzierung unternehmerischer F\&E und ein allzu forsches Inbeziehungsetzen von Fördervolumina zu Outputindikatoren ist auf der aggregierten Ebene wahrscheinlich nicht sehr vielsagend.

- Grundsätzlich werden auf Projektebene zwei Arten von Risiko relevant. Neben einem systematischen (d.h. exogenen - marktbezogenen) Risiko ist auch das idiosynkratische (d.h. endogene - technologiespezifische) zu berücksichtigen. Ursachen für systemische Risikokomponenten von F\&E-Projekten können z. B. Konjunkturschwankungen, Input-Preisänderungen allgemeiner technischer Fortschritt, oder auch sich ändernde Wettbewerbsbedingungen sein. Also Risiken, welche von Unternehmen nicht oder kaum beeinflussbar und daher exogen vorgegeben sind. Demgegenüber sind idiosynkratische Risikoanteile projektspezifisch (oder auch unternehmensspezifisch) und resultieren etwa aus der Ungewissheit über das Realisieren eines Entwicklungs- oder Erkenntnisprozesses im Zuge eines 
FTE-Projektes oder auch aus sehr viel »banaleren « Gründen, wie z.B. die Kündigung eines F\&E-Projektleiters. Diese Art von Risiko ist leichter einschätzbar, wenngleich beide Arten von Risiken miteinander in Zusammenhang stehen.

In diesem Zusammenhang ist beispielsweise die Arbeit der FFG-Basisprogramme viel weiter gediehen als die Systemevaluierung zu hoffen wagt. Denn dort wird seit langem jenes Bewertungsschema modifiziert, welches vor allem die technische und wirtschaftliche Qualität von Forschungsprojekten bewertet. Das antragstellende Unternehmen wird dahingehend geprüft, ob es förderwürdig ist (d. h. ob zum Beispiel Insolvenzgefahr vorliegt) und von welcher Größe heraus es den Antrag stellt. Wie schwierig eine Analyse über die Wirkungen der eingesetzten Fördermittel auf die gesamte unternehmerische Performanz ist, zeigen hingegen die ex-post Projektevaluierungen. Das einzige was daraus entsteht, sind irreführende Hebelzahlen.

Bei einem durchschnittlichen Finanzierungsanteil von 6,1\% kann nicht erwartet werden, dass Unternehmen besonders riskante Projekte zur Förderung einreichen. Die Unternehmen wissen, dass öffentliche F\&E-Förderung vor allem als Belohnung für innovative Unternehmen verstanden wird. Die beliebten success-stories der Förderagenturen lassen auch kaum einen anderen Schluss zu.

Statt die Erhöhung des Risikos auf Projektebene zu fordern, sollte sich der Staat auf jenen Bereich konzentrieren, wo Risiko per definitionem vorhanden ist: in der frühen Phase von Unternehmensgründungen im Hochrisikobereich. Sämtliche in der Systemevaluierung vorgeschlagenen Maßnahmen sind hier zu unterstützen und sollten sehr bewusst antizyklisch eingesetzt werden. Denn die derzeitige Krise schlägt vor allem auf den Wagniskapitalmarkt durch mit all seinen negativen Auswirkungen auf das Gründungsgeschehen. Die notwendigen Maßnahmen reichen von einem Kapitalmarktstärkungs- und Innovationsgesetz, das auch von der Community akzeptiert wird, über die Mobilisierung von Wagniskapital für die Frühphasenfinanzierungen durch eine direkte Beteiligung an Venture Capital-Fonds (Fund of Fund) bis hin zu einer Neugestaltung der Kapitalgarantien. Gleichwohl in Österreich ein feines System an Unterstützungsmaßnahmen existiert, liegt die Vermutung nahe, dass es ein noch zu hebendes Potential gibt. Hier drängt sich auch ein Unterscheidungsmerkmal der beiden Agenturen auf: hier die aws mit dem Instrumentenportfolio der Unternehmensgründungen und -wachstumsphasen und dort die FFG mit Projektförderungen sowie den thematischen und strukturellen Programmlinien. Dieses Unterscheidungsmerkmal bietet schon genug Raum für Bereinigung.

\section{Man lache nicht über die Follower}

Die Systemevaluierung führt eine Reihe von Unterscheidungsmerkmalen zwischen Ländern an der »technological front « und den »followers « an. Angeführt wird u. a. das Verhältnis zwischen radikalen und inkrementellen Innovationen, sowie dem Anteil der High-tech und wissensintensiven Sektoren an der gesamten Beschäftigung, Wertschöpfung und Exporten (S. 130). Auf beide Merkmale soll kurz eingegangen werden.

Für die Entwicklung radikaler Innovationen spielt F\&E eine (unter mehreren) entscheidende Rolle. Die erfolgreiche F\&E-Tätigkeit sichert nicht nur technologische Kompetenz, sondern verspricht auch langfristig höheren Unternehmenserfolg. Dies gilt insbesondere dann, wenn aus der eigenen F\&E marktfähige Produkte entspringen, die sich gegenüber Konkurrenzangeboten deutlich absetzen. Dadurch können zum einen Marktanteile gewonnen bzw. völlig neue Kundengruppen für das Unternehmen erschlossen werden. Dies ist die entscheidende Grundlage für ein rasches Wachstum von (kleinen) Unternehmen. Zum anderen entgehen die Unternehmen mit solchen Neuerungen einem Preiswettkampf auf reifen Märkten und können höhere Gewinnmargen erzielen. Gerade radikale Innovationen, also Marktneuheiten, die gleichzeitig neue Geschäftfelder öffnen, ist am ehesten ein solches Wachstums- und Gewinnpotential zuzuschreiben. Diese Innovationen bergen allerdings auch das größte Risiko, dass das Vorhaben scheitert. Sie überschreiten eine Technologieschwelle und gleichzeitig muss ein Vermarktungskonzept für eine neue Zielgruppe erarbeitet werden. Unternehmen, die diese Art der Produktinnovation hervorbringen, betreten bei Technologie und Markt Neuland und sind tendenziell mit der höchsten Unsicherheit und den höchsten Kosten konfrontiert.

Gleichzeitig sind mit der Entwicklung radikaler Innovationen auch Auswirkungen auf die Kompetenz eines Unternehmens zu erwarten. Auch wenn zu vermuten ist, dass Unternehmen, die selbst radikale Innovationen hervorbringen oder auf radikale Innovationen der Konkurrenz reagieren können, eine solide Kompetenzbasis haben, können radikale Innovationen eigenes Know-how entwerten - zumindest in dem unmittelbar betroffenen Bereich des Unternehmens (vgl. Christensen 1997).

Inkrementelle Innovationen hingegen sind laut OECDDefinition Innovationen, die an bestehenden Produktkonzepten anknüpfen, diese verbessern, ausdehnen oder ergänzen, und weder neu für den Markt noch neu im Sortiment des Unternehmens sind. Trotz des unüberhörbaren Appells, Österreich benötige mehr radikale Innovationen, darf die Herausforderung und der Vorteil einer »Follower «-Stratgie insbesondere für KMU nicht unterschätzt werden.

KMU sind in ihrem betrieblichen Alltag davon geprägt, dass Informationen unvollständig, Kompetenzen 


\section{Das österreichische Forschungsförderungssystem: Systemevaluiert}

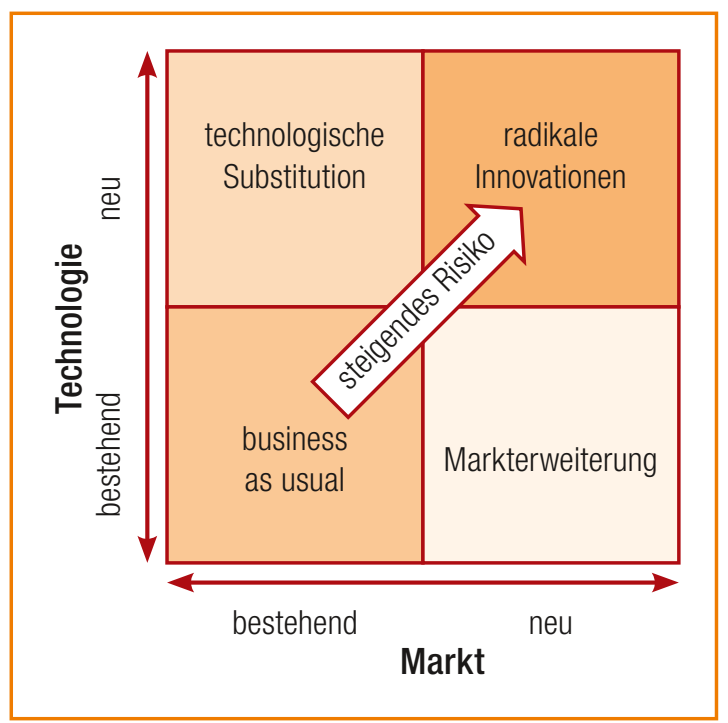

Zusammenhang Markt- und Technologie

Quelle: eigene Darstellung in Anlehnung an Hartmann, Myers (2000)

beschränkt und Ereignisse nur bedingt vorhersehbar sind. Allzu ambitionierte und zu weit nach vorne gerichtete Zielsetzungen, die in der Organisation des Unternehmens langfristige Weichenstellungen erfordern und Ressourcen binden, sollten vor diesem Hintergrund gesehen werden. Der Weg der kleinen, inkrementellen Anpassungen ist in diesem Kontext gerade für kleine und mittlere Unternehmen eine durchaus sinnvolle Alternative, die der radikalen Neuausrichtung des Unternehmens vorgezogen wird. Dieses Strategieverständnis setzt darauf, dass man bereit ist, seine strategische Orientierung im Lichte neuer Informationen und Erkenntnisse ständig zu überdenken und kontinuierlich anzupassen. Unternehmen sollten realisierbare Ziele für einen überschaubaren Zeitraum formulieren, ihre unternehmerischen Aktivitäten einer ständigen Reflexion unterziehen und evaluieren, damit bei Bedarf eine schnelle Anpassung an die neuen Gegebenheiten und Herausforderungen stattfinden kann (vgl. Tidd et al. 2001).

Für das strategische und operative Management von Innovation bedeutet die "Strategie der inkrementellen Schritte « (Spielkamp und Rammer 2004), dass es kein einfaches, dauerhaftes Standardkonzept in der Steuerung des Innovationsprozesses gibt. Situationen sind einmalig und Vorgänge in der Regel nicht reproduzierbar, sodass Wissen und Erfahrung natürlich helfen, aber ein unreflektierter Einsatz bewährter Instrumente, insbesondere der Glaube an allgemeingültige »best practices « und »Erfolgsfaktoren «
Gefahren beinhaltet. Unternehmen müssen daher darauf hinwirken, dass sie eine Routine in der Veränderung und in der Wandlungsfähigkeit erhalten. Dadurch wird tendenziell das Kompetenzprofil gestärkt, da man die vorhandene Produkt- und Technologiebasis nutzt - wenngleich durch das geringere Risiko auch die potentiellen Wettbewerbsvorteile nur in einem geringeren Umfang erzielt werden.

Grundsätzlich sehen sich Unternehmen also einem Trade-off zwischen einem deutlich sichtbaren, radikalen Bruch im Vergleich zu Produktentwicklungen der Vergangenheit dar (mit hohen Gewinnpotentialen, bei ebenfalls hohem Scheiterrisiko sowie hohen Innovationskosten) und eher inkrementellen, schrittweisen Verbesserungen, die mit geringeren Gewinnmöglichkeiten, aber auch mit geringeren Risiken und Kosten verbunden sind, gegenüber. Beide Strategien können erfolgversprechend sein, was eine für alle gleichermaßen gültige Frontrunnerstrategie ein wenig relativiert. Auch sollte sich langsam die Tatsache durchgesetzt haben, dass der österreichische Beitrag zum globalen technologischen Wandel ca. 1 \% beträgt und trotz der exponierten Position einiger Unternehmen, Österreich als Ganzes notwendigerweise immer ein follower-country sein wird.

\section{Sektorspezifika}

Auch das zweite Unterscheidungsmerkmal zwischen frontrunnern und followers birgt die Möglichkeit der Verwirrung in sich. Erstens, weil das gesamte F\&E-Fördersystem auf Förderung von Unternehmen (bzW. anderer Forschungsorganisationen) ausgerichtet ist. Unternehmen und nicht Sektoren werden gefördert. Und daher ist es einem Unternehmen, welches wächst und erfolgreich ist und mitunter auch eine staatliche F \&E-Förderung erhält, auch herzlich egal, welchem Sektor es zugerechnet wird.

Zweitens zeigen Sektoren, welche meist nach der durchschnittlichen F\&E-Intensität in high-, medium- und low-tech Sektoren unterteilt werden, eine zunehmende Heterogenität und beinhalten jeweils einen Mix von high-, medium- und low-tech Unternehmen. Eine ausschließlich sektorspezifische Betrachtung gerät in Gefahr, statistische Artefakte zu produzieren (Schibany et al. 2007). Der Sektoransatz überdeckt die Unterschiede auf Firmenebene. Heute spricht man von » innovation modes « (Jensen et al. 2007) um der komplexen und heterogenen Natur von Innovation sowie deren Verbreitung gerecht zu werden. Robertson und Patel (2007) hatten jüngst auf die Durchlässigkeit und Interdependenz wohldefinierter Sektoren hingewiesen. ${ }^{5}$ Die

\footnotetext{
5 "There is a reciprocal and close relationship between low-, medium- and high-tech sectors in developed economies which is crucial for overall economic success. Therefore, the levels of performance in low-, medium- and high-tech sectors are highly interdependent, resulting from reciprocal connections. Not only do innovations generated in high-tech sectors diffuse into sectors with lower levels of R\&D intensity, but low- and medium-tech firms are also involved in knowledge-creating activi-ties in high-tech fields. (Robertson und Patel 2007, siehe auch Kirner et al. 2009).
} 
folgende Abbildung zeigt am Beispiel Deutschlands die signifikante intra-sektorelle Heterogenität in Bezug auf die F\&E-Intensität der Unternehmen. Sie zeigt die Diskrepanz zwischen einer sektorspezifischen Klassifikation und der Realität auf Unternehmensebene: Im Schnitt erfüllen nur ca. die Hälfte der Unternehmen das sektorspezifische Kriterium bezüglich F \&E-Intensität.

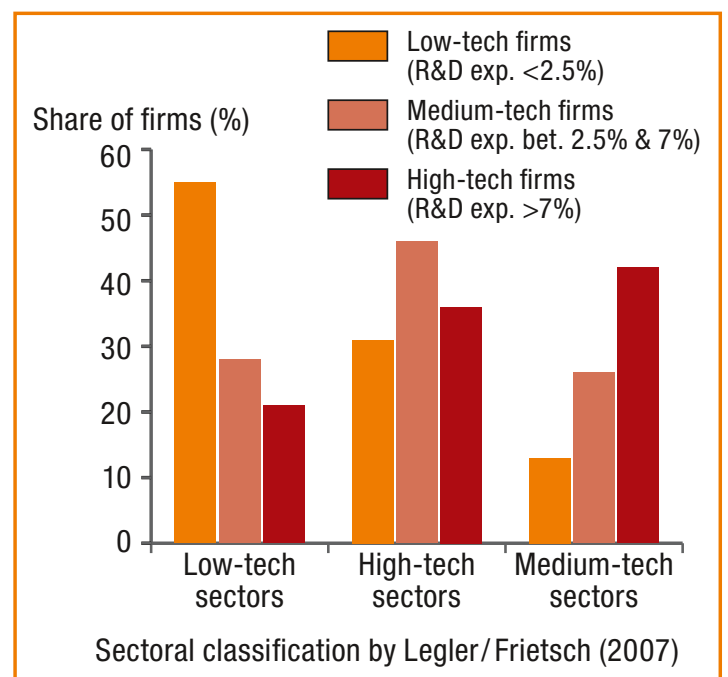

Quelle: Kirner et al. (2009)

Sektorbasierte Anteilsrechnungen sind somit kein wirklich aussagekräftiger Indikator für Wettbewerbsfähigkeit und Positionierung eines ganzen Landes an der »technological front«. Gleichwohl liefert die Arbeit an neuen Taxonomien - wie im Synthesebericht eindrucksvoll dargelegt - wichtige und neue Indizien für strukturellen Wandel und Dynamik. Und darum geht es schließlich - nur sollte der Strukturwandel möglichst breit verstanden werden.

Und allergrößte Vorsicht ist geboten, wenn die Verteilung von Fördervolumina nach Branchenaggregaten versucht wird. Denn auch hier überdeckt die Tücke der statistischen Konvention so manche Heterogenität und es kann leicht zu fehlgeleiteten Schlussfolgerungen kommen. So rechnen sich beispielsweise die Kplus Zentren als $\mathrm{GmbH}$ dem Dienstleistungsbereich zu, die beteiligten Unternehmenspartner stammen aber durchwegs aus dem Sachgüterbereich. Letztlich fließen somit auch die nicht unbeträchtlichen Fördervolumina aus diesem Strukturprogramm letztlich in die Industrie. Um wirklich die Kohärenz des Instrumentenmix (Teilbericht 8) beurteilen zu können, darf die Mikroebene nicht unberücksichtigt bleiben.

Ein weiteres Beispiel ist auch das fördertechnische Konstrukt des sogenannten »kooperativen Sektors «, der neben den Kplus Zentren, den beiden großen außeruniversitären Forschungseinrichtungen auch sämtliche Mitglieder der ACR umfasst. Dass darunter auch die AVL List, mit einem jährlichen Forschungsvolumen von ca. 82 Mio. $€$, fällt (mit all den Konsequenzen für die statistischen Durchschnittswerten) macht es notwendig, diesen Sektor nicht als eine »homogene « Einheit zu betrachten. ${ }^{6}$

\section{Abschließende Bemerkung}

Wie schon eingangs erwähnt, kann ein kurzer Kommentar und Diskussionsbeitrag niemals dem immensen Unterfangen einer Systemevaluierung gerecht werden. Die Systemevaluierung hat das gesamte österreichische Forschungs- und Fördersystem durchanalysiert - da blieb kein blinder Fleck übrig. Und die Betonung, dass es vor allem die Rahmenbedingungen sind, welche über den Erfolg und letztlich auch über die Zukunft entscheiden, ist den Evaluatorlnnen nicht hoch genug anzurechnen. Dass in einem kurzen Kommentar einige der Ergebnisse und Empfehlungen diskutiert und vielleicht andere Schlüsse gezogen werden, ist somit nur ein Hinweis, wie dynamisch und facettenreich dieses Politikfeld ist. Und letztlich liegt es an der Politik, aus diesen Debatten die richtigen Schlüsse zu ziehen. Da sind sich die Evaluatorlnnen und Kommentatoren wohl einig.

6 Mit dem Sonderfall AVL List lässt sich auch der überraschend hohe Anteil an Auslandsfinanzierung des kooperativen Sektors erklären. Der Großteil der Forschungsarbeiten wird im Auftrag internationaler Konzerne durchgeführt und damit finanziert. 


\section{Das österreichische Forschungsförderungssystem: Systemevaluiert}

\section{Referenzen}

Aiginger, K., R. Falk, A. Reinstaller (2009), Reaching out to the Future Needs Radical Change. Towards a New Policy for Innovation, Science and Technology in Austria, WIFO. Sämtliche Teilstudien befinden sich auf der Homepage des BMVIT: http://www.bmvit.gv.at/ innovation/forschungspolitik/systemevaluierung/index. html

Christensen, C. (1997), The Innovators Dilemma. When New Technologies Cause Great Firms to Fail, Boston.

CREST Expert Group Report (2008), "Policy Mix Peer Reviews: Country Report Austria", A Report of the CREST Policy Mix Expert Group, European Union Scientific and Technical Research Committee.

Europäische Kommission (2006), Wege zu einer wirksamen steuerlichen Förderung von FuE; KOM(2006) 728 endgültig.

Jensen, M., B. Johnson, E. Lorenz, B. A. Lundvall (2007), Forms of knowledge and modes of innovation. Research Policy 36, 680-693.

Kirner, E., S. Kinkel, A. Jaeger (2009), Innovation paths and the innovation performance of low-technology firms - An empirical analysis of German industry. Research Policy 38, 447-458.

Legler, H., R. Frietsch (2007), Neuabgrenzung der Wissenswirtschaft. Forschungsintensive Industrien undwissensintensive Dienstleistungen (NIW/ISI Listen 2006. Studien zum deutschen Innovationssystem Nr. 22, Bundesministerium für Bildung und Forschung (BMBF).

Hartmann, G., M. Myers (2000), Technical Risk, Product Specifications, and Market Risk, in: NIST (2000), Managing Technical Risk: Understanding Private Sector Decision Making on Early Stage, Technologybased Projects, ATP.

OECD (2007), Economic Surveys: Austria; Paris.

Robertson, P. L., P. R. Patel (2007), New wine in old bottles: technological diffusion in developed economies. Research Policy 36, 708-721.

Schibany, A., H. Gassler, G. Streicher (2007), High Tech or Not Tech. Vom fehlenden Strukturwandel und anderen Sorgen, InTeReg Working Paper Nr. 35 - 2007, JOANNEUM RESEARCH.

Schneider, H., W. Lueghammer, J. Schindler (2005), International Good Practices in der steuerlichen F\&E-Förderung. Unter besonderer Berücksichtigung junger und innovativer Unternehnen; IWI und JOANNEUM RESEARCH im Auftrag des BMWA.

Spielkamp, A., Ch. Rammer (2006),

Balanceakt Innovation - Erfolgsfaktoren im Innovationsmanagement kleiner und mittlerer Unternehmen, ZEW Dokumentation Nr. 06-04, Mannheim.

Tidd, J., J. Bessant, K. Pavitt (2001), Managing Innovation, 2. Aufl., Chichester 